\title{
Robust Filter Design Technique to Limit Resonance in Long Cables Connected to Power Converters
}

\author{
Tamiris Grossl Bade ，James Roudet ，Jean-Michel Guichon ，Patrick Kuo-Peng ， \\ and Carlos A. F. Sartori , Senior Member, IEEE
}

\begin{abstract}
A novel numerical analysis to characterize and to visualize the resonance behavior in a long cable is proposed. The resonance-due maximum amplification, and the frequency and position to which the maximum occurs, are represented in function of the load connected to the cable terminal in a 3-D plot here denominated "resonance surface response." This analysis is based on a white box modeling and allows the design of protective measures against the resonance phenomenon, such as filter design, protection against cross-talk, adequate voltage insulation choice, etc. One of these applications is exemplified: the design of an output filter for a power converter connected to a long cable, from which the results are compared to a classical design of the same filter to highlight the advantages and demonstrate the robustness of the proposed technique.
\end{abstract}

Index Terms-Embedded system design, power cable, resonance in long cable, white-box model.

\section{INTRODUCTION}

$\mathbf{R}$ ESONANCE in long cables is a recurrent problem in the literature, principally in applications where long power cables are connected to power converters, e.g., connections between motors and its drives [1]-[4], the feeding cable of high-speed trains [5], connections between offshore generator stations, and onshore electrical substations [6]-[8]. The resonance occurs because medium-to-high frequency harmonics are generated by the switched converters and circulate in unshielded long cables with mismatched impedances.

Indeed, the input impedance values for power converters are not likely to be close to the characteristic impedance of the cable. The power cables used in power conversion or industrial systems, as the aforementioned applications, have non-negligible losses, can easily be very long, and are not always shielded. For lossy cables connected to a generic load, a complete analytical description of the resonance behavior, i.e., the resonance frequency and resonance-due amplification, is difficult to obtain. In

Tamiris Grossl Bade, James Roudet, and Jean-Michel Guichon are with the University Grenoble Alpes, CNRS, Grenoble INP, G2Elab, 38000 Grenoble, France (e-mail: tamirisgbade@ gmail.com; james.roudet@g2elab.grenobleinp.fr; jean-michel.guichon@g2elab.grenoble-inp.fr).

Patrick Kuo-Peng is with the Federal University of Santa Catarina, Florianópolis 88040-900, Brazil (e-mail: patrick.kuo.peng@ufsc.br).

Carlos A. F. Sartori is with the Escola Politécnica da Universidade de São Paulo, São Paulo 05508-010, Brazil, and with the Instituto de Pesquisas Energéticas e Nucleares, São Paulo 05508-900, Brazil (e-mail: sartori@ pea.usp.br). this article, a numerical approach able to describe the resonance behavior of any multiconductor cable is detailed. This is an innovative approach, where the information of the resonance behavior of the cable is represented in the form of a multidimensional surface denominated "resonance surface response."

To exemplify the utility of this new representation of resonance, the design of an electromagnetic interference (EMI) filter for the conducted emissions of a power converter connected to a long cable using the resonance surface response is proposed in this article.

Typically, EMI filters are designed to meet international standards, such as the CISPR 11 for industrial applications or the DO-160 for airborne devices, all of which determine that the conducted emissions are measured with a line impedance stabilization network (LISN) [9]-[13].

Naturally, the device behaves differently when connected to the actual system, because the input impedance of a complex network (which varies considerably with frequency) can be drastically different from the LISN impedance. From another perspective, the interaction between the reactance of the network and the reactive elements of the filter alter its behavior, inducing new resonances that amplify the stray signals [14]. Therefore, the standards do not guarantee the good performance of the device when connected to a real network, particularly if the network includes long cables.

For embedded systems requiring power converters, such as aircraft or electric vehicles, it is possible to design an output filter taking into account the behavior of the system, as the whole network is known to the developers. To design filters taking all the possible resonances into account contributes to the robustness of the filter, as shown in [9] and[14] where the cable was modeled by a series inductance. This is an acceptable model for a frequency band to which the wavelength is considerably smaller than a quarter of the cable length $(\lambda \ll \ell / 4)$. However, the power converter conducted emissions often reach frequencies where this condition is not satisfied, and only a long cable model can predict the multiple resonance frequencies and positions.

The resonance surface response proposed in this article allows a filter design based on the complete transmission-line model that can be classified as a white box modeling, because the exact behavior of the complete system is taken into account. The power cables taken into account in this study have per-unit-length (p.u.1.) parameters that vary considerably with frequency. For that reason, both the surfaces and the simulations presented here were calculated in the frequency domain, using a simulator 


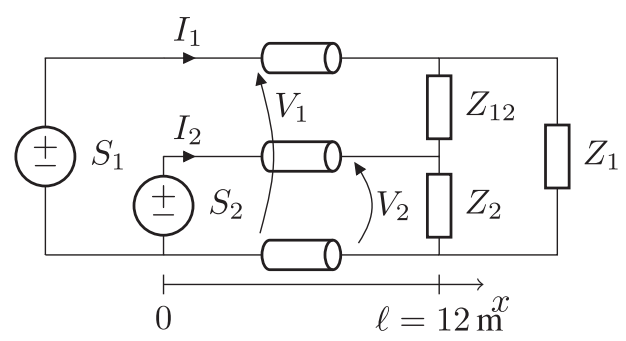

Fig. 1. Schematic of the cable for the resonance study.

coded into MATLAB. This simulator allows the most accurate representation of the cable frequency-dependent parameters, as it demands no approximations whatsoever. It has been validated experimentally with a resonant cable setup in [15].

The resonance surface response of a three-conductor power cable is introduced in Section II, and in Section III, it is applied to the design of an EMI filter for conducted emissions and compared to a classical design method.

\section{SuRface Response of CABle Resonance}

The resonance of voltage and current in a long cable is a well-known phenomenon, with potential to cause damage. It is avoidable by matching the terminal impedances with the characteristic impedance of the line. However, in power systems, impedance matching is rarely possible and the resonance phenomenon must be taken into account in the design process of a system with medium-to-high frequency EMI parasitic signals. Indeed, if $\ell$ is the length of the longer cable in the system and $\lambda$ the shortest wavelength circulating through it, resonance will occur if $\ell>\lambda / 4$.

The analytical determination of the resonance frequency and amplification in a lossy line connected to a generic load is not a trivial task, needing the solution of implicit nonlinear equations. For that reason, a numerical analysis of the resonance behavior of a cable is proposed.

This analysis is based on the solution of (1)-(7) [16], able to represent the system in Fig. 1

$$
\begin{aligned}
{\left[\begin{array}{c}
\mathbf{V}(x) \\
\mathbf{I}(x)
\end{array}\right]=\left[\begin{array}{ll}
\boldsymbol{\Phi}_{1}(x) & \boldsymbol{\Phi}_{2}(x) \\
\boldsymbol{\Phi}_{3}(x) & \boldsymbol{\Phi}_{4}(x)
\end{array}\right]\left[\begin{array}{c}
\mathbf{V}(0) \\
\mathbf{I}(0)
\end{array}\right] } \\
\boldsymbol{\Phi}_{1}(x)=\mathbf{Z}_{c} \cosh (x \sqrt{\mathbf{Y Z}}) \mathbf{Y}_{\mathbf{c}} \\
\boldsymbol{\Phi}_{2}(x)=-\mathbf{Z}_{c} \sinh (x \sqrt{\mathbf{Y Z}}) \\
\mathbf{\Phi}_{3}(x)=-\sinh (x \sqrt{\mathbf{Y Z}}) \mathbf{Y}_{\mathbf{c}} \\
\mathbf{\Phi}_{4}(x)=\cosh (x \sqrt{\mathbf{Y Z}}) \\
\mathbf{V}(0)=\left[\begin{array}{l}
S_{1} \\
S_{2}
\end{array}\right] \\
\mathbf{I}(\ell)=\mathbf{Y}_{L} \mathbf{V}(\ell) .
\end{aligned}
$$

In (1)-(5), $x$ is the space axis parallel to the line, $\ell$ is the cable length, $\mathbf{I}$ and $\mathbf{V}$ are vectors of size $n$ containing, respectively, the currents of each active conductor and the voltages relative to the reference conductor; $\mathbf{Z}_{c}=\mathbf{Y}^{-1} \sqrt{\mathbf{Y Z}}$ is the characteristic

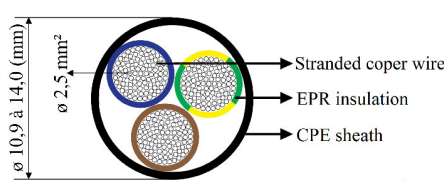

Fig. 2. $\quad 2.5 \mathrm{~mm}^{2}$ three-conductor cable cross section.

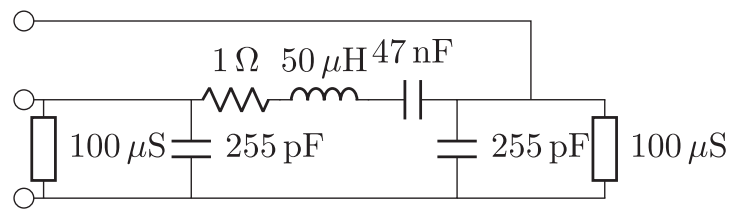

Fig. 3. Load used as example, modal input impedance: $Z_{L e}$.

impedance matrix, $\mathbf{Y}_{c}=\mathbf{Z}_{c}^{-1}$ is the characteristic admittance matrix, and $\sqrt{\mathbf{Y Z}}$ is the nondiagonalized propagation matrix. Matrices $\mathbf{Z}=\mathbf{R}+j \omega \mathbf{L}$ and $\mathbf{Y}=\mathbf{G}+j \omega \mathbf{C}$ are the p.u.l. impedance and admittance matrices, respectively. The definition of the p.u.l. resistance $\mathbf{R}$, inductance $\mathbf{L}$, conductance $\mathbf{G}$, and capacitance $\mathbf{C}$ matrices can be found in [16]; $j$ is the imaginary unity and $\omega$ is the angular frequency. In (7), $\mathbf{Y}_{L}$ is the input admittance matrix representing a generic equivalent circuit of the devices connected to the cable terminal [17].

The frequency-domain simulator used along this document consists in the solution of (1)-(7) for each element of a discretized frequency vector $\omega[n]$. This simulator is detailed and its results are experimentally validated in [15]. In the system presented in Fig. 1, the three-phase load composed by $Z_{1}, Z_{2}$, and $Z_{12}$ can represent any passive device [17].

The resonance behavior of the system shown in Fig. 1 can be described in function of the real and imaginary parts of the load impedances. However, as three impedances are necessary to represent a generic load and the real and imaginary parts of each one of them have to be considered as individual variables, the analysis would result in a 7-D surface: 2-D for each impedance and one to present the results would be needed. Such a surface cannot be plotted, even though the numerical analysis is feasible.

Because the goal of this article is to present a visual representation of resonance behavior of a three-conductor transmission line, the analysis is limited to balanced systems, i.e., with decoupled common and differential modes. The definition of differential and common modes are given

$$
\begin{array}{ll}
V_{\mathrm{DM}}=V_{1}-V_{2} & I_{\mathrm{DM}}=\frac{I_{1}-I_{2}}{2} \\
V_{\mathrm{CM}}=\frac{V_{1}+V_{2}}{2} & I_{\mathrm{CM}}=I_{1}+I_{2} .
\end{array}
$$

In a balanced system, the modes can be analyzed individually. For the system to be balanced, two conditions need to be respected: $Z_{1}=Z_{2}$, and the three-conductor cable has to be circular symmetric [18]. This is the case of the three-conductor cable "Nexans 3G2.5 Titanex," its cross section is presented in Fig. 2. The p.u.l. parameters of this cable have been experimentally identified in function of the frequency with an impedance 
TABLE I

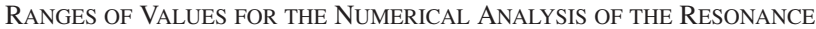

\begin{tabular}{cccc}
\hline Variable & Range & Number of points & Scaling \\
\hline$A_{D M}, A_{C M}$ & {$[0,100] \mathrm{k} \Omega$} & 401 & $\log$ \\
$B_{D M}, B_{C M}$ & {$[-100,100] \mathrm{k} \Omega$} & 401 & $\log$ \\
$f$ & {$[0.01,10] \mathrm{MHz}$} & 5000 & $\log$ \\
$x$ & {$[0,12] \mathrm{m}$} & 25 & linear \\
\hline
\end{tabular}

analyzer as described in [15] and[19]. The parameters of the cable are not included here, they are plotted in [15, Fig. 7].

As the parameters have been identified in function of the frequency and a frequency-domain simulation is used, the cable losses are accurately represented for the whole frequency band. This feature is important to guarantee the accuracy of the resonance amplification and frequency [15].

The load impedance in differential and common modes, with $Z_{1}=Z_{2}$, are, respectively, defined as

$$
\begin{aligned}
& Z_{L_{\mathrm{DM}}}=\frac{2 Z_{1} Z_{12}}{Z_{12}+2 Z_{1}} \\
& Z_{L_{\mathrm{CM}}}=\frac{Z_{1}}{2} .
\end{aligned}
$$

The numerical analysis to build the resonance surface response consists in performing the frequency-domain simulation of the system for different values of $Z_{L_{\mathrm{DM}}}=A_{\mathrm{DM}}+j B_{\mathrm{DM}}$ and $Z_{L_{\mathrm{CM}}}=A_{\mathrm{CM}}+j B_{\mathrm{CM}}$, for the whole frequency band at different positions along the line, and by saving the maximum voltage amplification $V(x) / V(0)$ and its frequency and position for each point $(A, B)$. The range and number of points in the discretization of each of these variables are resumed in Table I.

The maximum amplification voltage, the resonance frequency, and the position along the cable where the resonance takes place are plotted in function of the load in Fig. 4 for the differential mode and in Fig. 5 for the common mode. All figures are plotted with $A$ and $-B$ in a $\log$ scale, the surface for positive $B$ is not represented here but can be found in [20, page 80].

The voltage amplification surfaces represent the whole set of the highest voltage amplification for each load impedance. The case of matched impedances is in the area where $B$ is low and $A_{\mathrm{DM}} \approx 80 \Omega$ in Fig. 4(a) or $A_{\mathrm{CM}} \approx 60 \Omega$ in Fig. 5(a): in these conditions, the amplification is 1 because resonance does not occur. This effect introduces discontinuities in the frequency and position resonance surfaces.

Outside the matched impedance zone, two tendencies of the surfaces can be highlighted, for both modes. The first is for high values of $A$ and $B$ : in this case, the amplification tends to 16 times the input at a resonance frequency of $3.1 \mathrm{MHz}$, taking place at $x=\ell$. This behavior is similar to the resonance in the open-ended cable, case in which the resonance frequency is $f_{\mathrm{OC}}=v / 4 \ell \approx 3.13 \mathrm{MHz}$ (propagation speed in the cable: $v \approx 150 \times 10^{6} \mathrm{~m} / \mathrm{s}$ ), and where the maximal voltage occurs necessarily at $x=\ell$, because $I(\ell)=0$.

The second tendency appear for low values of $A$ and $B$, where the amplification tend to be 8.8 for a resonance of frequency $6.3 \mathrm{MHz}$ taking place at $x=\ell / 2$. This behavior is similar to a short-circuited cable, of which the resonance frequency is $f_{\mathrm{SC}}=$ $v / 2 \ell \approx 6.25 \mathrm{MHz}$ taking place at the cable midpoint.

The voltage amplification is higher for high $A$ and $B$ because the resonance frequencies are lower and, as a consequence, the cable losses are smaller. For the same reason, the highest resonance amplification does not occur for the open-ended cable, but for a capacitive load (peak of 16.8).

To exemplify one possible analysis on these surfaces position along the cable where the resonance takes place are plotted in function of the load in Fig. 4 for the differential mode and in Fig. 5 for the common mode. All figures are plotted with $A$ and $-B$ in a $\log$ scale, the surface for positive $B$ is not represented here but can be found in [20, page 80].

The voltage amplification surfaces represent the whole set of the highest voltage amplification for each load impedance. The case of matched impedances is in the area where $B$ is low and $A_{\mathrm{DM}} \approx 80 \Omega$ in Fig. 4(a) or $A_{\mathrm{CM}} \approx 60 \Omega$ in Fig. 5(a): in these conditions, the amplification is 1 because resonance does not occur. This effect introduces discontinuities in the frequency and position resonance surfaces.

Outside the matched impedance zone, two tendencies of the surfaces can be highlighted, for both modes. The first is for high values of $A$ and $B$ : in this case, the amplification tends to 16 times the input at a resonance frequency of $3.1 \mathrm{MHz}$, taking place at $x=\ell$. This behavior is similar to the resonance in the open-ended cable, case in which the resonance frequency is $f_{\mathrm{OC}}=v / 4 \ell \approx 3.13 \mathrm{MHz}$ (propagation speed in the cable: $v \approx 150 \times 10^{6} \mathrm{~m} / \mathrm{s}$ ), and where the maximal voltage occurs necessarily at $x=\ell$, because $I(\ell)=0$.

The second tendency appear for low values of $A$ and $B$, where the amplification tend to be 8.8 for a resonance of frequency $6.3 \mathrm{MHz}$ taking place at $x=\ell / 2$. This behavior is similar to a short-circuited cable, of which the resonance frequency is $f_{\mathrm{SC}}=$ $v / 2 \ell \approx 6.25 \mathrm{MHz}$ taking place at the cable midpoint.

The voltage amplification is higher for high $A$ and $B$ because the resonance frequencies are lower and, as a consequence, the cable losses are smaller. For the same reason, the highest resonance amplification does not occur for the open-ended cable, but for a capacitive load (peak of 16.8).

To exemplify one possible analysis on these surfaces, the modal input impedances $Z_{L e}$ of the load shown in Fig. 3 has been plotted in red, superposed with the frequency surfaces. The intersection points between the line representing $Z_{L e}$ and the resonance surface response correspond to the resonance frequency of the cable connected to $Z_{L e}$. The resonance amplification and position can be determined by taking the equivalent point in the amplification and position response surfaces. These intersections are marked with black rectangles in Figs. 4 and 5. These intersections show that the differential-mode resonance causes a 15.2 amplification at $2.92 \mathrm{MHz}$, and the common mode a 14.3 amplification at $2.29 \mathrm{MHz}$, both taking place at $x=\ell$.

One can imagine several applications for these surfaces: filter design aiming to reduce dangerous resonance amplifications, mitigation of EMI phenomenon such as crosstalk, the adequate choice of the critical voltage of the insulation of the cable (seen that resonance can considerably amplify the system nominal voltage), among others. 


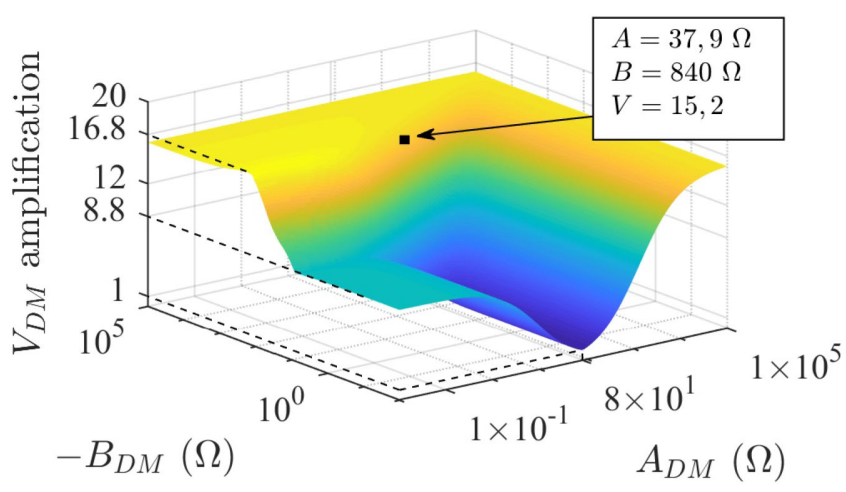

(a)

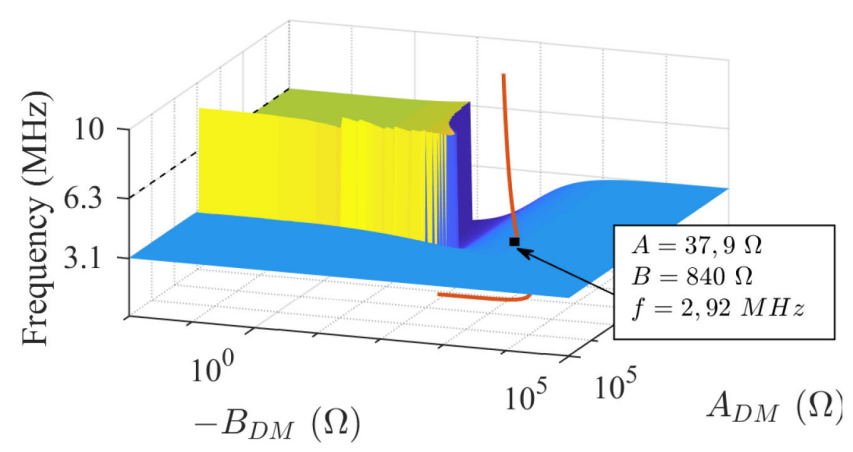

(b)

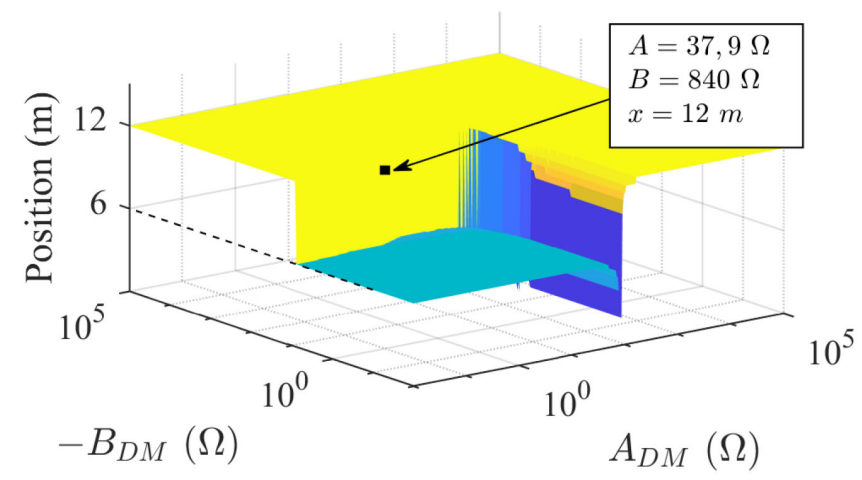

(c)

Fig. 4. Differential-mode resonance behavior of the cable in function of $Z_{L_{\mathrm{DM}}}$; in red: $Z_{L e}$, markers: intersection with $Z_{L e}$. (a) Voltage amplification. (b) Resonance frequency. (c) Resonance position.

\section{APPliCATION: Filter DESIGN FOR POWER CONVERTER}

A theoretical case study was chosen to demonstrate the utility of the resonance surface response: the design of a passive output filter for a power converter in a known system. The theoretical system for this study is presented in Fig. 6.

It consists in a buck converter powered by a battery through a long three-conductor cable. The conductors work as phase, neutral, and protective earth (PE) of the system, the PE is connected to the device chassis. The cable is the same used in Section II, its cross section is presented in Fig. 2. The cable length of $30 \mathrm{~m}$ was chosen to ensure that the cable is long enough for multiple resonances to be on the switching harmonics

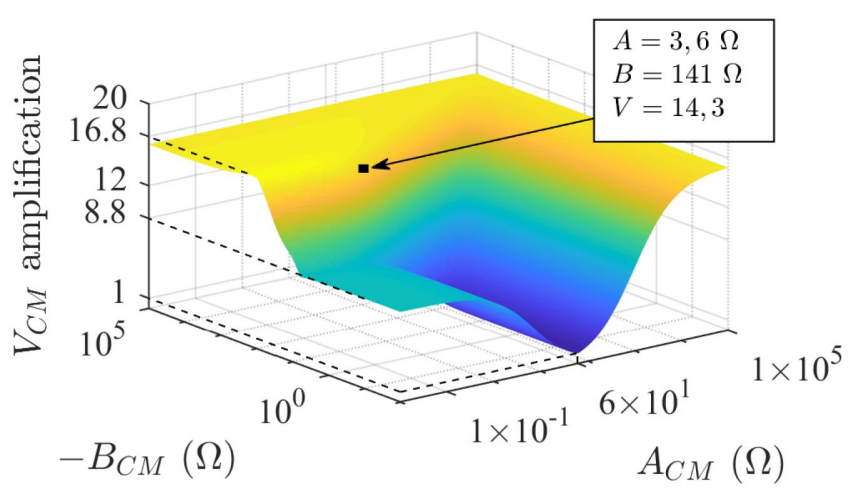

(a)

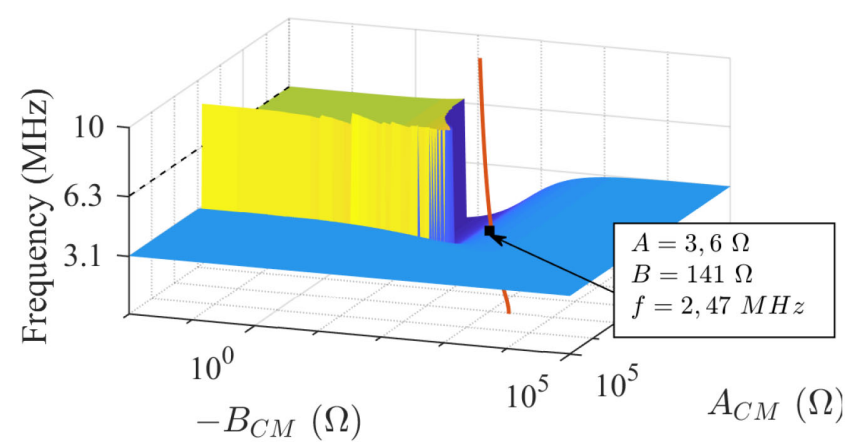

(b)

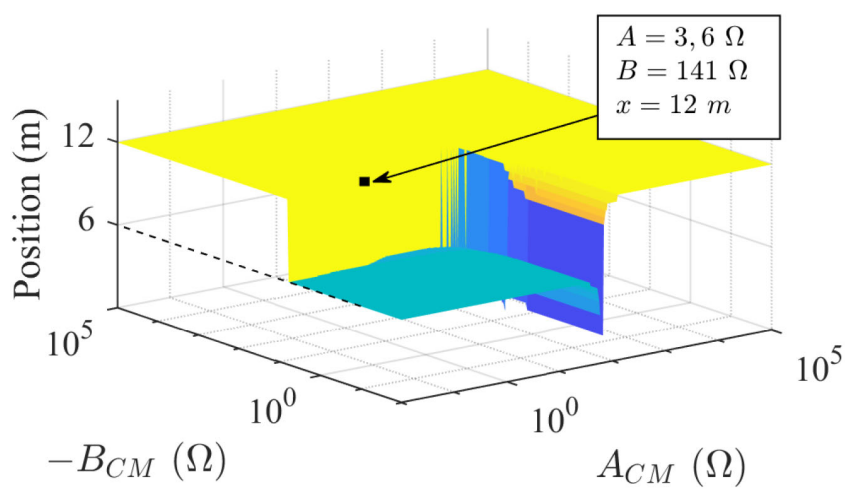

(c)

Fig. 5. Common-mode resonance behavior of the cable in function of $Z_{L_{\mathrm{CM}}}$; in red: $Z_{L e}$, markers: intersection with $Z_{L e}$. (a) Voltage amplification. (b) Resonance frequency. (c) Resonance position.

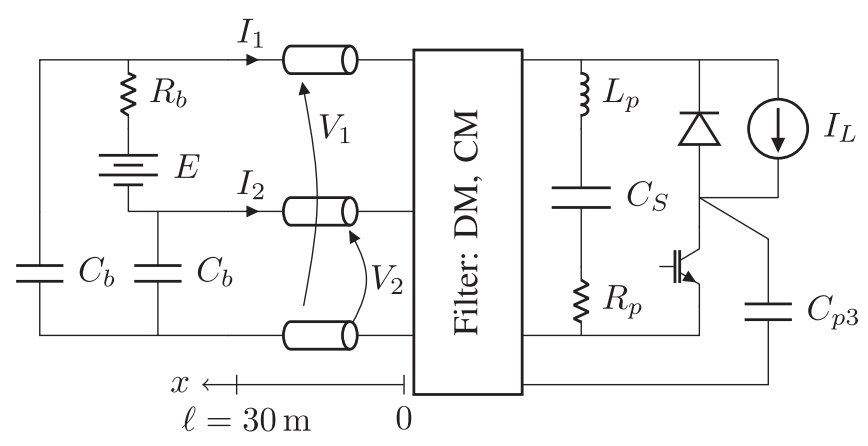

Fig. 6. System for EMI filter design. 


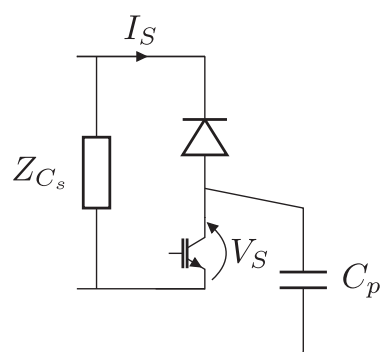

Fig. 7. Switching-cell model.

frequency band but still short enough for the attenuation due to the cable losses not to be too high.

This study is performed using the aforementioned frequencydomain simulator, coded in MATLAB. The power converter is represented in the frequency domain using the model proposed in [21], which substitutes the switching cell by equivalent trapezoidal sources (cf., Figs. 7 and 8). Specifically, the association of this converter model and the long cable model introduced in the previous section is experimentally validated in [15].

Because the results of the frequency-domain simulator have been scientifically validated for a configuration very similar to the one chosen in this section, the simulator can be used to demonstrate the effectiveness of the filter design technique derived from the resonance frequency response.

The battery is modeled with stray capacitances $C_{b}$ representing the electrical connection to its chassis. The structure of the buck converter was simplified by replacing its load by a dc current source to neglect the output current ripple, what does not impact the medium-to-high frequency harmonics generated by the switching cell. The values of the parameters in the schematics of Fig. 6 are in Table II.

The output filter has the structure shown in the schematic in Fig. 9. It consists in a common-mode filter composed by $C_{y}, L_{\mathrm{CM}}$ close to the switching cell, followed by a differentialmode filter composed by $C_{x}, L_{\mathrm{DM}}$ close to the device output. This is a common filter structure in power converters [9].

For comparison ends, the filter elements will first be designed with a classical approach (see Section III-A), and then with the proposed resonance surface response (see Section III-B).

For this example, a conducted emission limit was chosen based on the standard RTCA DO-160G. The filter design will be based on numerical simulations [9], [13], [15]. The conducted emission limit is the extent of application of the aforementioned standard: its measurement conditions are not reproduced on the simulations presented here.

\section{A. Example of Classical Filter Design Technique}

A good guide for what is here called the "classic" design approach is presented in [13]. In a few words, the classical approach consists in the calculation of the necessary attenuation to be imposed by the filter, by subtracting from the device output currents the limit of conducted emissions stipulated by the standard. Once the required attenuation is known, the filter is designed to meet it. The validation of the resultant filter is

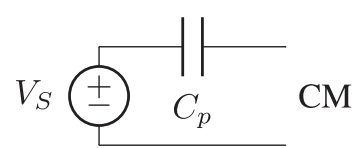

(a)

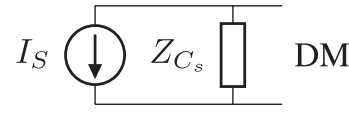

(b)

Fig. 8. Switching-cell model. (a) Common mode. (b) Differential mode.

TABLE II

Numeric Values of the System Parameters; $f_{s}$ IS THE SWITChING FREQUENCY, $t_{r}$ RISE TIME, AND $t_{f}$ FALL Time

\begin{tabular}{cc|cc|cc|cc}
\hline$C_{S}$ & $10 \mu \mathrm{F}$ & $C_{p 3}$ & $100 \mathrm{pF}$ & $C_{b}$ & $200 \mathrm{pF}$ & $t_{r_{V_{S}}}$ & $100 n s$ \\
$L_{p}$ & $20 \mathrm{nH}$ & $I_{L}$ & $10 \mathrm{~A}$ & $R_{b}$ & $50 \mathrm{~m} \Omega$ & $t_{f_{V_{S}}}$ & $200 n s$ \\
$R_{p}$ & $10 \mathrm{~m} \Omega$ & $E$ & $400 \mathrm{~V}$ & $f_{s}$ & $20 \mathrm{kHz}$ & $t_{r_{I_{S}}}$ & $80 n s$ \\
& & & & & & $t_{f_{I_{S}}}$ & $120 n s$ \\
\hline
\end{tabular}

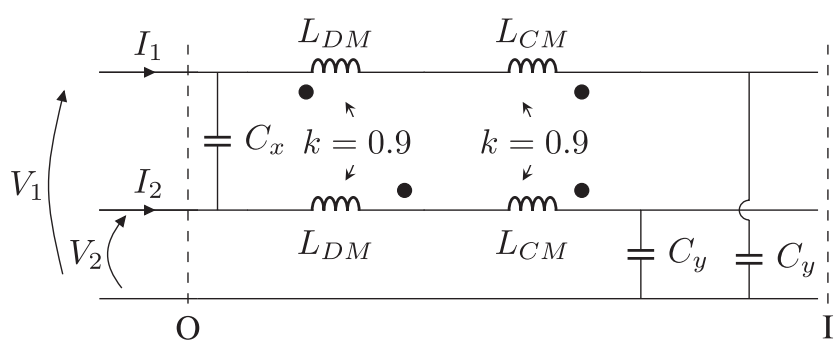

Fig. 9. EMI filter structure; to the left O: output and to the right I: input.

TABLE III

Filter Designed With Classical Approach

\begin{tabular}{l|ll}
\hline $\mathrm{CM}$ & $C_{y}=68 \mathrm{nF}$ & $L_{C M}=107 \mu \mathrm{H}$ \\
$\mathrm{DM}$ & $C_{x}=22 \mathrm{nF}$ & $L_{D M}=23.5 \mu \mathrm{H}$ \\
\hline
\end{tabular}

performed under the conditions of a compliance test, with the device connected to an LISN.

This method was applied to the system in Fig. 6. The conducted emissions of the switching device without filter were estimated with a frequency-domain simulation [15], supposing the device is connected standard impedances of $50 \Omega$ (ideal LISN). The simulations are performed for the discrete set of frequencies multiple of the switching frequency.

The filter elements designed with the classical approach following [13] are resumed in Table III. The adequacy of the designed filter is verified with a frequency-domain simulation [15] of the current that would be measured by a LISN, for each harmonic frequency. The results are plotted in Fig. 10 along with the chosen conducted emission limit. The results show the filter meets the standard constraints.

However, the classical method does not guarantee that the conducted emission limit will be respected when the buck converter is connected to the actual system. Indeed, by performing the same simulation with the converter connected to the 30-m-long three-conductor cable, the device output currents 


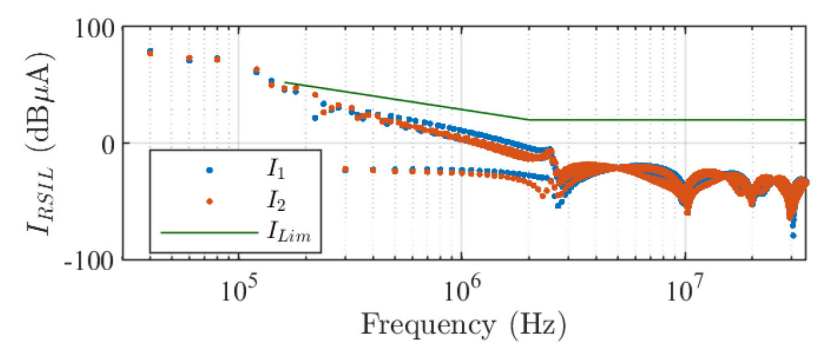

Fig. 10. Filter output current when connected to an LISN.

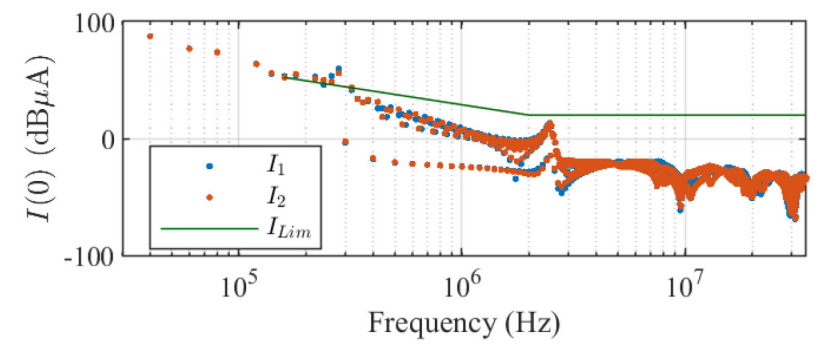

Fig. 11. Filter output current when connected to the 30-m cable (cf., Fig. 6).

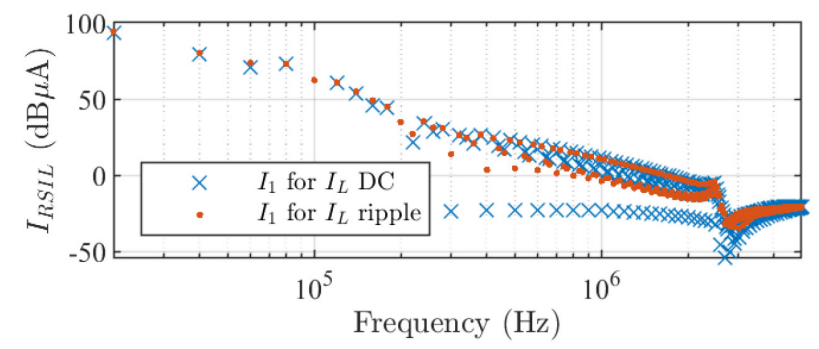

Fig. 12. Approximation of $I_{L}$ dc is valid (cf., Fig. 6), simulation of filter connected to $50 \Omega$ loads; blue crosses: $I_{L}=10 \mathrm{~A}$, red dots: $I_{L}=10 \mathrm{~A} \pm 50 \%$ ripple.

exceed the standard limit, as shown in Fig. 11. The association of the filter and the long cable introduces an extra resonance to the system, causing an unpredicted amplification of the conducted emissions [14].

Therefore, to improve the robustness of the system, it would be better to take the behavior of the complete system into account in the filter design. Moreover, the harmonics injected at the cable input, in Fig. 11, can be amplified by the resonance phenomena in the long cable, reaching even higher values somewhere else along the cable. The design using the resonance surface response solves both of these uncertainties: it guarantees that the conducted emissions will respect a stipulated limit anywhere along the cable.

To validate the hypothesis that the load connected to the buck converter can be represented by a dc current source, an extra simulation was performed: $I_{1}$ was calculated supposing that the load absorbs a current $I_{L}$ with a ripple of $\pm 50 \%$ around its dc value. The results are plotted along with $I_{1}$ from Figs. 10 to 12 . It can be concluded that the ripple on $I_{L}$ has a negligible effect in the input current harmonics.

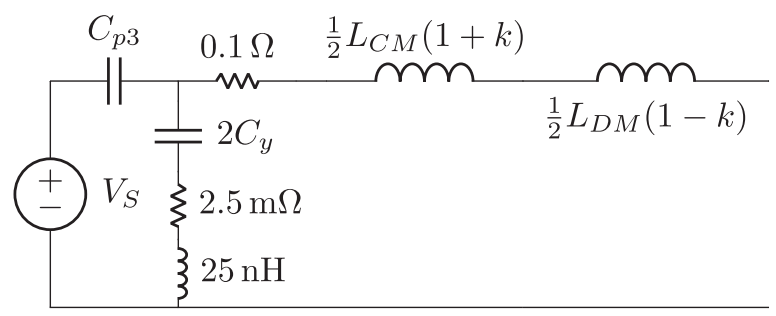

Fig. 13. Buck converter equivalent circuit in common mode.

\section{B. Resonance-Based Design Technique}

The resonance surface response presented in Section II can predict the maximal amplification of stray signals anywhere along the cable taking the exact behavior of the cable into account. This tool will now be used to design a filter and the surfaces, which can be plotted in function of any two real variables, will be plotted in function of the filter element so that the filters can be easily designed from them. The proposed method guarantees that the harmonics will respect a limit of emissions anywhere along the cable in spite of resonance-due amplifications.

Two sets of surfaces will be calculated for differential and common modes, the definition of the modes are given in (8) and (9), using the frequency-domain simulator. It is important to recall that an analysis with coupled modes is possible but not presentable in a 3-D space, and that the frequency-domain simulator used in this section has been experimentally validated in a former paper [15].

The chosen frequency-domain models of the power converter in each mode were proposed in [21], and are shown in Figs. 7 and 8. A circuit analysis of the filter in Fig. 9 gives the following equations linking the output voltages and currents (subscript $O$ ) to the input voltages and currents (subscript $I$ ) in common and differential modes:

$$
\begin{aligned}
V_{\mathrm{DM}_{O}}= & \left(1+\frac{Z_{L_{\mathrm{DM}}}}{Z_{C_{y}}}\right) V_{\mathrm{DM}_{I}}-2 Z_{L_{\mathrm{DM}}} I_{\mathrm{DM}_{I}} \\
I_{\mathrm{DM}_{O}}= & -\frac{2 Z_{L_{\mathrm{DM}}}+2 Z_{C_{x}}+Z_{C_{y}}}{2 Z_{C_{y}} Z_{C_{x}}} V_{\mathrm{DM}_{I}} \\
& +\left(1+\frac{2 Z_{L_{\mathrm{DM}}}}{Z_{C_{x}}}\right) I_{\mathrm{DM}_{I}} \\
V_{\mathrm{CM}_{O}}= & \left(1+\frac{Z_{L_{\mathrm{CM}}}}{Z_{C_{y}}}\right) V_{\mathrm{CM}_{I}}-\frac{1}{2} Z_{L_{\mathrm{CM}}} I_{\mathrm{CM}_{I}} \\
I_{\mathrm{CM}_{O}}= & -\frac{2}{Z_{C_{y}}} V_{\mathrm{CM}_{I}}+I_{\mathrm{CM}_{I}}
\end{aligned}
$$

with

$$
\begin{aligned}
& Z_{L_{\mathrm{DM}}}=j \omega\left(L_{\mathrm{DM}}(1+k)+L_{\mathrm{CM}}(1-k)\right) \\
& Z_{L_{\mathrm{CM}}}=j \omega\left(L_{\mathrm{DM}}(1-k)+L_{\mathrm{CM}}(1+k)\right) .
\end{aligned}
$$

From (12) to (17), the complete common-mode and differentialmode circuit models of the buck converter and its filter can be built, and they are drawn in Figs. 13 and 14, respectively. In these schematics, the parasitic elements of the filter were taken 


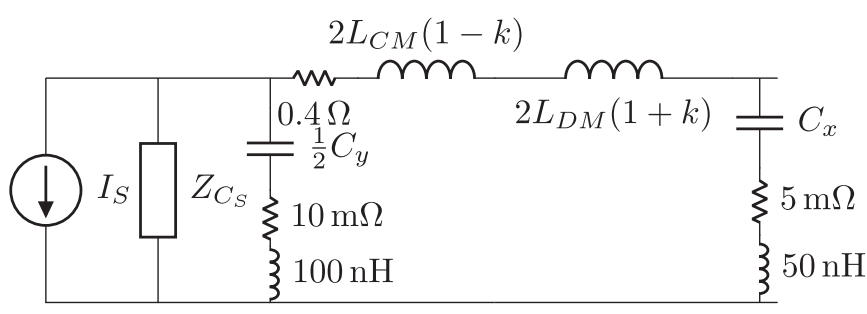

Fig. 14. Buck converter equivalent circuit in differential mode.

TABLE IV

PARAmeters For Resonance Surface CALCUlation

\begin{tabular}{c|ccc}
\hline & Value range & Number of points & Scale \\
\hline$f$ & {$[150 \mathrm{kHz}, 47.5 \mathrm{MHz}]$} & 3000 & $\log$ \\
$x$ & {$[0,30 \mathrm{~m}]$} & 31 & linear \\
$C_{y}, C_{x}$ & {$[0.1 \mathrm{nF}, 100 \mathrm{nF}]$} & 400 & $\log$ \\
$L_{C M}, L_{D M}$ & {$[1 \mu \mathrm{H}, 10 \mathrm{mH}]$} & 401 & $\log$ \\
\hline
\end{tabular}

into account because they have an impact the attenuation of high-frequency harmonics.

These models were coded into the frequency-domain simulator [15], the trapezoidal sources $V_{S}$ and $I_{S}$ being represented by their Fourier Series. From this model, the surfaces were plotted in function of $C_{y}$ and $L_{\mathrm{MC}}$ in common mode and $C_{x}$ and $L_{\mathrm{MD}}$ in differential mode.

The frequency band in which the amplification were calculated starts at $150 \mathrm{kHz}$, the minimum frequency to which most standards apply, up to $3.5 / t_{r_{m}}$, where $t_{r_{m}}$ is the minimum rise/fall time in the system. In this case, we considered $t_{r_{m}}=80 \mathrm{~ns}$, what gives an upper limit for the frequency band of $47.5 \mathrm{MHz}$. The currents are calculated along the cable. The ranges of values to which the simulations were performed are resumed in Table IV, along with the number of points and their scaling.

The common-mode surface will be plotted first, because the only impact of the differential-mode filter in the common-mode equivalent circuit is an extra inductance of value $L_{\mathrm{DM}}(1-k)$, as shown in Fig. 13, while for the system in differential mode, the series association of the common-mode filtering capacitances $C_{y}$ has a stronger impact.

In common mode, variations on the value of $L_{\mathrm{DM}}$ have only $5.2 \%$ of the impact of variations on $L_{\mathrm{CM}}$, as shown below

$$
\frac{\frac{\partial Z_{L_{\mathrm{CM}}}}{\partial L_{\mathrm{DM}}}}{\frac{\partial Z_{L_{\mathrm{CM}}}}{\partial L_{\mathrm{CM}}}}=\frac{1-k}{1+k}=5.2 \%
$$

For this reason, $L_{\mathrm{DM}}$ was fixed to its predesigned value $L_{\mathrm{DM}}=$ $23.5 \mu \mathrm{H}$ (cf., Table III) for common-mode simulations.

The complete representation of the system in common mode is shown in Fig. 13. The system was simulated for all the combination of variable values given in Table IV. The pairs $\left(L_{\mathrm{CM}}, C_{y}\right)$ to which the cutoff frequency of the filter $1 / \sqrt{(1+k) L_{\mathrm{CM}} C_{y}}$ was bigger than $150 \mathrm{kHz}$ were not taken into account, because for these pairs, the filter would not attenuate the first harmonics in the frequency band.

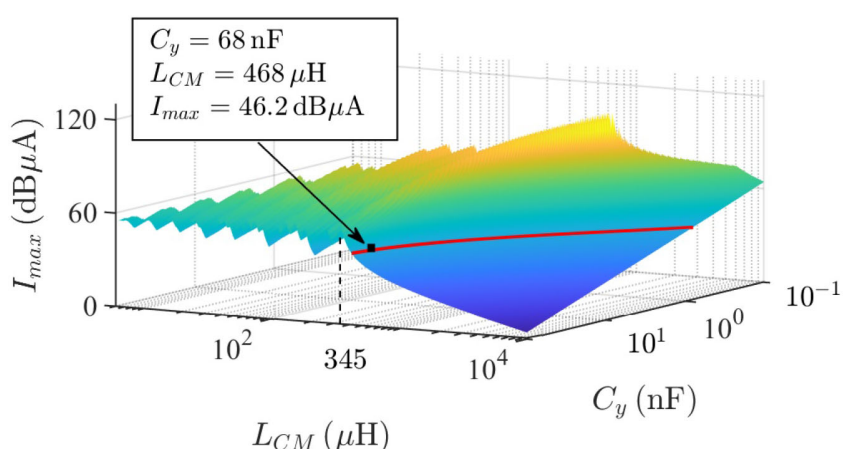

Fig. 15. Maximum common-mode conducted emissions along the cable in function of $C_{y}$ and $L_{\mathrm{CM}}$; intersection with limit in red.

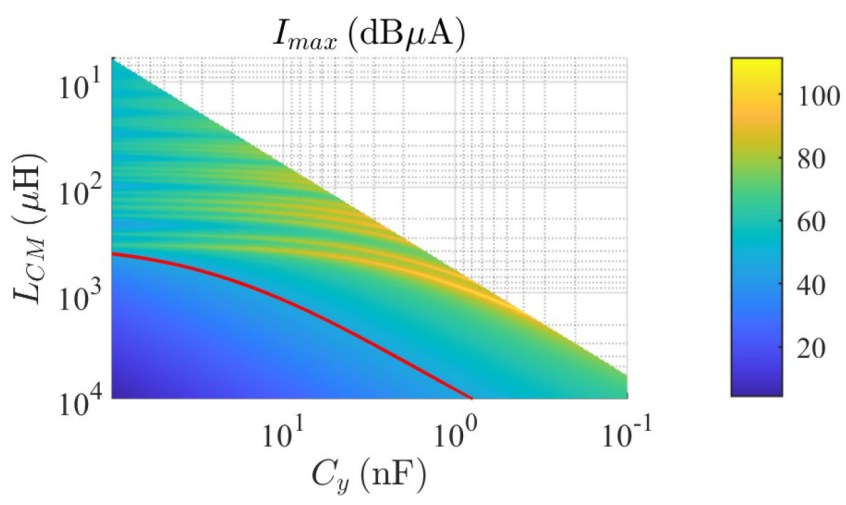

Fig. 16. Top view of the surface in Fig. 15.

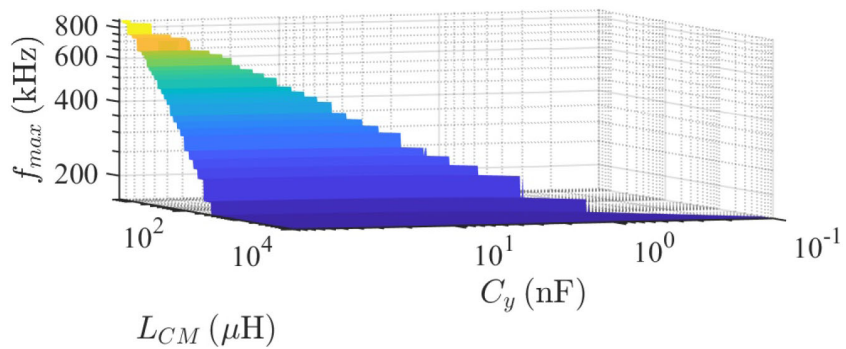

Fig. 17. Frequencies of the maximum in common mode.

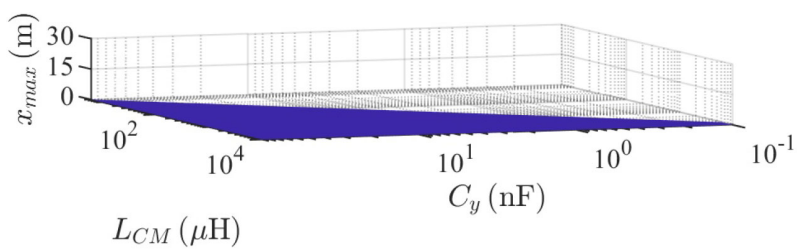

Fig. 18. Position of the maximum in common mode.

The highest current harmonic level along the cable in the whole frequency band is plotted in function of the commonmode filter elements in Fig. 15, and its top view is in Fig. 16. The frequencies and position to which they occurred are plotted in Figs. 17 and 18. 


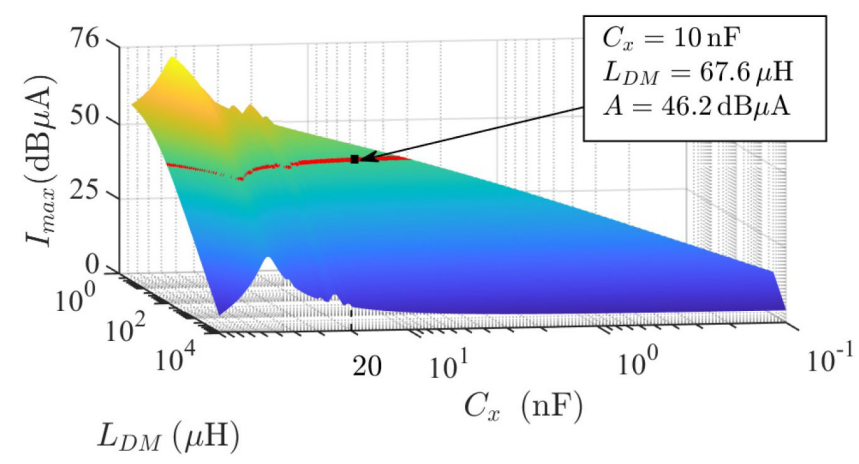

Fig. 19. Maximum differential-mode conducted emissions along the cable in function of $C_{x}$ and $L_{\mathrm{DM}}$; intersection with limit in red.

Oscillations can be seen in the surface of Fig. 15 for $L_{\mathrm{CM}}<$ $345 \mu \mathrm{H}$. They are due to a new resonance between the cable and $L_{\mathrm{CM}}$. In Fig. 17, it can be seen that the frequency of this resonance increases when $L_{\mathrm{CM}}$ decreases. The cable has an inductive behavior in these frequencies and impacts the cutoff frequency of the complete system, which no longer depends mainly on $L_{\mathrm{CM}}$.

The values of the filter parameters should be chosen from the smooth zone in Fig. 15, so the possible inaccuracies engendered by an experimental realization would not cause a drastic change in the response of the system.

In this zone, the frequency to which the biggest harmonic occurs is $160 \mathrm{kHz}$, the first switching harmonic in the studied frequency band. For this frequency, the conducted emission limit converted to common mode is $46.2 \mathrm{~dB} \mu \mathrm{A}$. The zone in the smooth portion of the surface that corresponds to this value is marked in red in Fig. 15. The pair $\left(L_{\mathrm{CM}}, C_{y}\right)$ should be chosen from this line, and by setting $C_{y}=68 \mathrm{nF}$, the surface gives $L_{\mathrm{CM}}=468 \mu \mathrm{H}$.

These values $\left(C_{y}=68 n F, L_{\mathrm{CM}}=468 \mu \mathrm{H}\right)$ are used in the evaluation of the differential-mode resonance surface response. The complete system in differential mode is represented in Fig. 14. The simulations were performed for the values presented in Table IV.

The maximum current harmonics in the system for each pair $\left(C_{x}, L_{\mathrm{DM}}\right)$ are plotted in Fig. 19, the pairs $\left(C_{x}, L_{\mathrm{DM}}\right)$ to which the cutoff frequency of the filter $1 / \sqrt{2(1+k) L_{\mathrm{DM}} C_{x}}$ was bigger than $150 \mathrm{kHz}$ were not taken into account. Fig. 20 shows the top view of Fig. 19. The frequencies to which each maximum occurred are plotted in Fig. 21, and the position along the cable where it takes place is plotted in Fig. 22.

The maximum current harmonics in differential mode are considerably less important than in common mode, mainly because of the filtering effect of the capacitances $C_{S}$ and $C_{y}$.

The maximum amplification surface shown in Fig. 19 predicts higher harmonics for $C_{x}$ between 20 and $90 \mathrm{nF}$, therefore, it is better to choose $C_{x}<20 \mathrm{nF}$.

With this condition, the frequency of the highest harmonic is $160 \mathrm{kHz}$, and the conducted emission limit in differential mode is the same as in the common-mode case. The maximum harmonics around $46.2 \mathrm{~dB} \mu \mathrm{A}$ are highlighted in red in Fig. 19. With $C_{x}=10 \mathrm{nF}$, an inductance $L_{\mathrm{DM}}=67.6 \mu \mathrm{H}$ is sufficient.

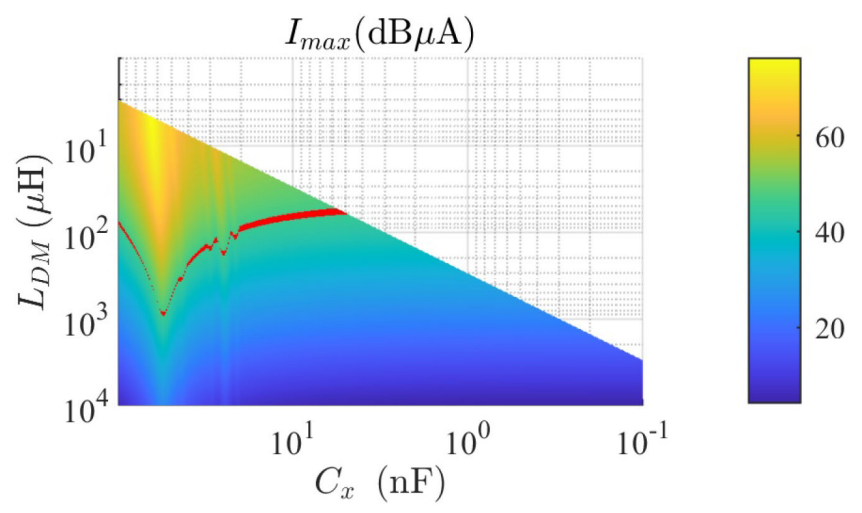

Fig. 20. Top view of the surface in Fig. 19.

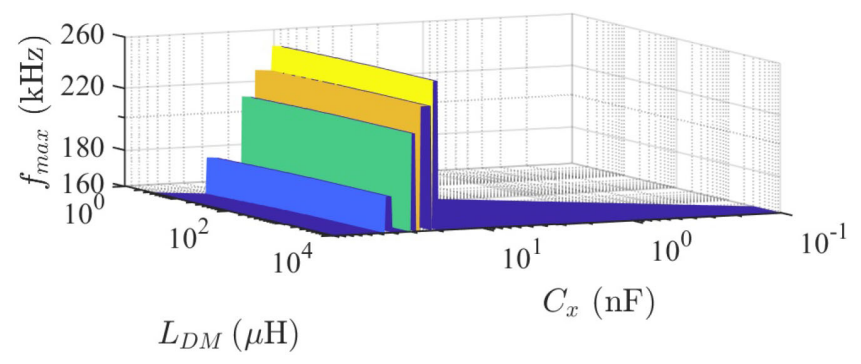

Fig. 21. Frequencies of the maximum in differential mode.

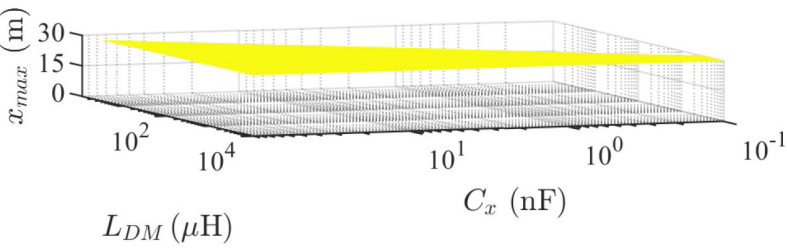

Fig. 22. Position of the maximum in differential mode.

TABLE V

Filter Designed With Resonance SuRface Response

\begin{tabular}{l|ll}
\hline $\mathrm{CM}$ & $C_{y}=68 \mathrm{nF}$ & $L_{C M}=468 \mu \mathrm{H}$ \\
$\mathrm{DM}$ & $C_{x}=10 \mathrm{nF}$ & $L_{D M}=67.8 \mu \mathrm{H}$ \\
\hline
\end{tabular}

The filter values designed with the resonance surface response are resumed in Table $\mathrm{V}$.

The currents at the cable input and output, the positions where the highest harmonic amplitudes take place as shown in Figs. 18 and 22 , were simulated with the frequency-domain simulator for the filter parameters of Table V. Their harmonics are plotted against the conducted emission limit in Fig. 23.

This figure shows that the proposed technique succeeds in designing a filter that respects the standard in both the positions in which the maximum current harmonics occurred (see Figs. 18 and 22). Table VI compares the agreement of the two filters (classic or resonance surface designed) with the conducted emission limit when the converter is connected to the long cable. With the surfaces, a $6 \mathrm{~dB} \mu \mathrm{A}$ security gap is respected, whereas, with the 


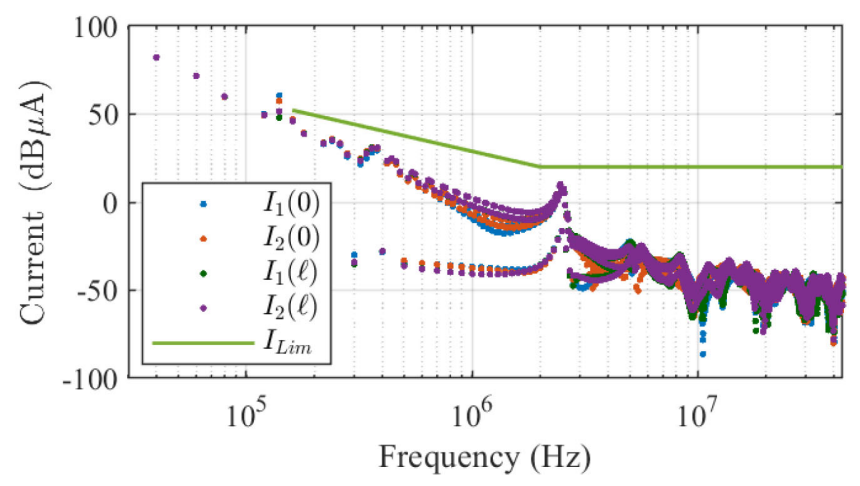

Fig. 23. Current harmonics at $x=0$ and $x=\ell$ with the filter designed from the resonance surfaces.

TABLE VI

COMPARISON OF DESIGNED FILTERS $[0.15,30] \mathrm{MHz}$

\begin{tabular}{c|cc}
\hline & $\max \left(I_{1}(0)\right)$ with cable & $I_{\mathrm{DO}-160}-\max \left(I_{1}(0)\right)$ \\
\hline Classic [13] & $59.55 \mathrm{~dB} \mu \mathrm{A}$ & $-14.47 \mathrm{~dB} \mu \mathrm{A}$ \\
Surfaces & $46.08 \mathrm{~dB} \mu \mathrm{A}$ & $6.13 \mathrm{~dB} \mu \mathrm{A}$ \\
\hline
\end{tabular}

classic design, the harmonics are $14.47 \mathrm{~dB} \mu \mathrm{A}$ higher than the standard limit.

This approach guarantees that the conducted emissions of the switching device will not exceed the standard limit anywhere along the cable, contributing to the robustness of the designed system. The technique proposed here would be well-adapted to embedded systems, because, in this case, the whole system is known by the developers.

\section{CONCLUSION}

This article proposes a new tool to analyze the resonance behavior of a long cable in function of the impedances connected to its terminals: the "surface resonance response." These surfaces were plotted for balanced systems with three-conductor cables, in differential and common modes. For unbalanced systems, or systems with more than three conductors, these surfaces can still be calculated, even if they cannot be plotted in a 3-D space. These surfaces are useful to the design of robust systems, possibly avoiding critical configurations where the resonance phenomenon in a long cable may cause damage to the devices connected to it. An application example was proposed: the design of an EMI filter of a buck converter connected to a long cable in an embedded system. By taking the resonance behavior into account in the filter design, it is guaranteed that the conducted emissions respect the standard anywhere along the cable connected to the converter.

\section{REFERENCES}

[1] H. Akagi and I. Matsumura, "Overvoltage mitigation of inverter-driven motors with long cables of different lengths," IEEE Trans. Ind. Appl., vol. 47, no. 4, pp. 1741-1748, Jul./Aug. 2011.

[2] A. F. Moreira and T. H. Lipo, "High-frequency modeling for cable and induction motor overvoltage studies in long cable drivers," IEEE Trans. Power Electron., vol. 38, no. 5, pp. 1297-1306, Oct. 2002.

[3] H. De Paula, D. A. de Andrade, M. L. R. Chaves, J. L. Domingos, and M. A. A. de Freitas, "Methodology for cable modeling and simulation for high-frequency phenomena studies in PWM motor drives," IEEE Trans. Power Electron., vol. 23, no. 2, pp. 744-752, Mar. 2008.
[4] L. Wang, C. Ngai-Man Ho, F. Canales, and J. Jatskevich, "High-frequency modeling of the long-cable-fed induction motor drive system using TLM approach for predicting overvoltage transients," IEEE Trans. Power Electron., vol. 25, no. 10, pp. 2653-2664, Oct. 2010.

[5] X. Chu, F. Lin, and Z. Yang, "The analysis of time-varying resonances in the power supply line of high speed trains," in Proc. Int. Power Electron. Conf., 2014, pp. 1322-1327.

[6] S. Zhang, S. Jiang, X. Lu, B. Ge, and F. Z. Peng, "Resonance issues and damping techniques for grid-connected inverters with long transmission cable," IEEE Trans. Power Electron., vol. 29, no. 1, pp. 110-120, Jan. 2014.

[7] M. C. Sousounis, J. K. H. Shek, and M. A. Mueller, "Filter design for cable overvoltage and power loss minimization in a tidal energy system with onshore converters," IEEE Trans. Sustain. Energy, vol. 7, no. 1, pp. 400408, Jan. 2016.

[8] M. Kuschke and K. Strunz, "Transient cable overvoltage calculation and filter design: Application to onshore converter station for hydrokinetic energy harvesting," IEEE Trans. Power Del., vol. 28, no. 3, pp. 1322-1329, Jul. 2013.

[9] B. Touré, J. Schanen, and L. Gerbaud, "EMC modeling of drives for aircraft applications: Modeling process, EMI filter optimization, and technological choice," IEEE Trans. Power Electron., vol. 28, no. 3, pp. 1145-1156, Mar. 2013.

[10] M. Hartmann, H. Ertl, and J. W. Kolar, "EMI filter design for a $1 \mathrm{MHz}$, $10 \mathrm{~kW}$ three-phase/level PWM rectifier," IEEE Trans. Power Electron., vol. 26, no. 4, pp. 1192-1204, Apr. 2011.

[11] J. C. X. Zhang and C. Wen, "Harmonics attenuation and power factor correction of a more electric aircraft power grid using active power filter," IEEE Trans. Power Electron., vol. 63, no. 12, pp. 7310-7319, Dec. 2016.

[12] Y. Liu, D. Jin, S. Jiang, W. Liang, J. Peng, and C. Lai, "An active damping control method for the LLCL filter-based SiC MOSFET grid-connected inverter in vehicle-to-grid application," IEEE Trans. Veh. Technol., vol. 68, no. 4, pp. 3411-3423, Apr. 2019.

[13] K. Raggl, T. Nussbaumer, and J. W. Kolar, "Guideline for a simplified differential-mode EMI filter design,” IEEE Trans. Ind. Electron., vol. 57, no. 3, pp. 1031-1040, Mar. 2010.

[14] L. Zhai, X. Zhang, and N.E. A. Bondarenko, "Mitigation emission strategy based on resonances from a power inverter system in electric vehicles," Energies, vol. 9, no. 6, 2016, Art. no. 419.

[15] T. G. Bade et al., "Frequency-domain modeling of unshielded multiconductor power cables for periodic excitation with new experimental protocol for wide band parameter identification," Electr. Eng., vol. 101, pp. 333-343, 2019.

[16] C. Paul, Analysis of Multiconductor Transmission Lines. Piscataway, NJ, USA: IEEE Press, 2008.

[17] M. Hosoya, "The simplest equivalent circuit of a multi-terminal network," Bull. Fac. Sci., Univ. Ryukyus, vol. 70, pp. 1-10, 2000.

[18] A. Sugiura and Y. Kami, "Generation and propagation of common-mode currents in a balanced two-conductor line," IEEE Trans. Electromagn. Compat., vol. 54, no. 2, pp. 466-473, Apr. 2012.

[19] J. Knockaert, J. Peuteman, J. Catrysse, and R. Belmans, "A vector impedance meter method to characterize multiconductor transmission-line parameters," IEEE Trans. Electromagn. Compat., vol. 52, no. 4, pp. 10191025, Nov. 2010.

[20] T. G. Bade, "Characterization of the cabling on industrial power networks for EMI simulation," Ph.D. dissertation, , Université Grenoble Alpes, Giéres, France, 2019. [Online]. Available: https://hal.archives-ouvertes. fr/tel-02409933

[21] B. Revol, J. Roudet, J.-L. Schanen, and P. Loizelet, "EMI study of threephase inverter-fed motor drives," IEEE Trans. Ind. Appl., vol. 47, no. 1, pp. 223-231, Jan. 2011.

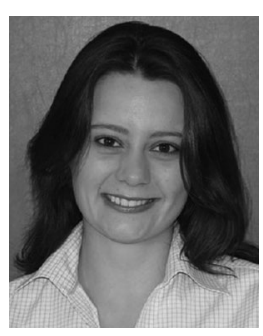

Tamiris Grossl Bade was born in Curitiba, Brazil. She received the B.Sc. degree, in 2016 and Ph.D. degree, in 2019, both in double-degree from the Federal University of Santa Catarina, Florianópolis, Brazil, with the Grenoble INP Institute, Grenoble, France).

She is currently a temporary-attached Teacher and Researcher with the Grenoble Electrical Engineering Laboratory, Grenoble INP Institute. Her research interests include radio-frequency EMC on power cables and power converters. 


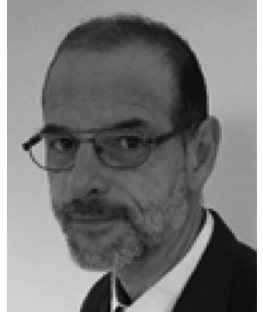

James Roudet was born in 1962. He both his engineering diploma and his Ph.D. from the Grenoble Institute of Technology, Grenoble, France, in 1986 and the Ph.D. degree, in 1990.

He is a Professor at Grenoble-Alpes University, Grenoble, France, and he was the Director of Grenoble Electrical Engineering Laboratory for 12 years. His research interests include the modeling of parasitic elements of power converter and systems, to improve layout and power design connections. He is a source of creation of numerical tools to take into account EMC effects for designing power converters and systems. He had also led the power electronics team for several years.

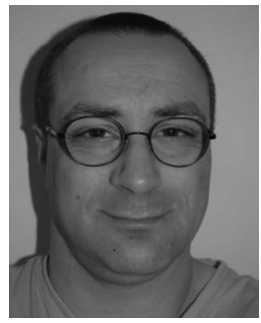

Jean-Michel Guichon received the diploma degree in electrical engineering and the Ph.D. degree from Grenoble Institute of Technology, Grenoble, France, in 1998 and 2001, respectively.

$\mathrm{He}$ is an Associate Professor with the Université Grenoble Alpes, Grenoble, France. He has been with the Grenoble Electrical Engineering Laboratory since 2003 , working in the field of electromagnetic modeling for power electronics. He research interests include the development of numerical tools to compute in low frequency (without propagation).
Patrick Kuo-Peng was born in Toamasina, Madagascar. He received the graduate degree in electrical engineering from the Université Paul Sabatier of Toulouse, Toulouse, France, in 1990, and the doctoral degree in power electronics from the Institut National Polytechnique de Toulouse, Toulouse, France, in 1993.

He is currently a Full Professor with the Department of Electrical Engineering, Universidade Federal de Santa Catarina, Florianópolis, Brazil. His special fields of interest include electromagnetic field calculations by numerical methods. He is the author or co-author of nearly 220 technical papers published in journals and conference proceedings.

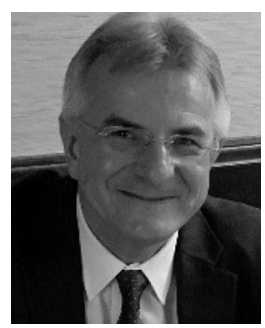

Carlos A. F. Sartori (Senior Member, IEEE) received the B.Sc., M.Sc., and the Ph.D. degrees from Escola Politécnica of University of São Paulo (EPUSP), São Paulo, Brazil, in 1983, 1994, and 1999, all of them in electrical engineering.

He was with the National Commission on Nuclear Energy CNEN/IPEN-SP (1984-2018), where he was the Technical Lead responsible of the Electromagnetic Compatibility (EMC) Group. He is an Invited Professor with the Graduated Programs of EPUSP (1999) and at the Nuclear and Energy Research Institute IPEN-CNEN/SP (2006), and an Associate Professor with the Catholic University of São Paulo (PUC/SP), where he was the Vice-Head of the Department of Electrical Engineering (1997-1998), and the Vice-Dean (2001-2004). His current research interests include applied electromagnetics and EMC.

Dr. Sartori is the South Brazil IEEE EMC Chapter Chair and a former IEEE EMC Board of Directors Member. He is also a Member of the International Compumag Society and of the Brazilian Society on Electromagnetics, where he was the Vice-President (1998-2002). He has co-authored more than 100 papers published in international journals and conference proceedings. 